\title{
RESULTS ON FINITE DENSITY QCD
}

Ian M. Barbour, Susan E. Morrison, ${ }^{\text {a }}$ Elyakum G. Klepfish, ${ }^{\mathrm{b}}$ John B. Kogut ${ }^{\mathrm{c}}$ and Maria-Paola Lombardo ${ }^{\mathrm{d}}$

${ }^{a}$ Dept. of Physics and Astronomy, University of Glasgow, G12 8QQ, U.K. UKQCD Collaboration

${ }^{b}$ Dept. of Physics, King's College London, London WC2R 2LS

${ }^{\mathrm{c}}$ Department of Physics, University of Illinois, 1110 West Green Street, Urbana, IL 61801

dZentrum für interdisziplinäre Forschung, Universität Bielefeld, D-33615 Bielefeld, Germany

A brief summary of the formulation of QCD at finite chemical potental, $\mu$, is presented. The failure of the quenched approximation to the problem is reviewed.

Results are presented for dynamical simulations of the theory at strong and intermediate couplings. We find that the problems associated with the quenched theory persist: the onset of non-zero quark number does seem to occur at a chemical potential $\approx \frac{m_{\pi}}{2}$. However analysis of the Lee-Yang zeros of the grand canonical partition function in the complex fugacity plane, $\left(e^{\mu / T}\right)$, does show signals of critical behaviour in the expected region of chemical potential.

Results are presented for a simulation at finite density of the Gross-Neveu model on a $16^{3}$ lattice near to the chiral limit. Contrary to our simulations of QCD no pathologies were found when $\mu$ passed through the value $\frac{m_{\pi}}{2}$.

\section{Introduction}

Lattice QCD at finite baryon density holds the key to an understanding of the phase transition between quark-confined hadronic matter and the quark-gluon plasma. At zero temperature we expect this transition to occur at $\mu=\frac{m_{p}}{3}$ corresponding to the lowest lying state with non-zero baryon number.

Although the QCD phase transition at finite temperature has been successfully studied on the lattice with simulations predicting a first order transition at a well defined critical temperature, a non-perturbative lattice calculation of nuclear matter at finite density has been an outstanding problem in QCD thermodynamics since 1986 11.2]. The fundamental difficulty in simulating QCD at finite density and investigating the transition quantitatively is that the effective action resulting from the Grassmann integration over the fermions is complex due to the introduction of the chemical potential in the Dirac matrix. This complex nature of the QCD finite density action[3] [3] prohibits the use of naive probabilistic methods in evaluating the functional integral. Thus the standard Hybrid Monte Carlo simulation algorithms for lattice QCD with dynamical fermions are inappropriate in this context since they require a positive definite probability measure.

The study of lattice QCD at finite density is motivated by the need for a full equation of state for nuclear matter in the temperature-chemical potential plane which would give quantitative predictions for critical energies and densities in relativistic heavy ion collisions. Other applications include equations of state for neutron stars and cosmological models of the early universe. 


\section{Quenched Simulations}

Serious problems were first reported in quenched simulations of finite density $\mathrm{QCD}$ in 1986 [1] and the physical and mathematical reasons for this failure have been the focus of considerable debate ever since [2,5].

In these early simulations the behaviour of the chiral condensate was studied at fixed quark mass for various different values of the chemical potential, $\mu$. The behaviour was initially as expected viz. the chiral condensate remained constant up until a certain value of mu and then tended to zero as the chemical potential was increased.

We would expect that physical observables are $\mu$ independent up to some $\mu_{c}$ which is related to the threshold for baryon production. The problem encountered was the following: as the quark mass was decreased the $\mu$ at which the chiral condensate began to change also decreased towards zero.

In fact, the onset of chiral symmetry restoration appeared to occur at a chemical potential of half the pion mass, which would extrapolate to zero in the chiral limit. A study of the distribution of the eigenvalues of the lattice Dirac operator confirmed that the lowest mass state containing a net number of fermions (i.e. a quark or a baryon but not a meson which does not see $\mu$ ) became massless as the bare quark mass was reduced to zero. The interpretation was that there existed either baryonic states which became massless in the chiral limit and had an energy equal to $\frac{3}{2} m_{\pi}$ or stable quark matter with low mass per baryon.

The result that $\mu_{c}$ is proportional to the pion mass is clearly unphysical.We expect $\mu_{c} \simeq \frac{m_{p}}{3}$ because the proton is the lightest state with nonzero baryon number. From this we are drawn to two possible conclusions. The first possibility is that the quenched approximation is at fault so that it is strictly necessary to consider the complex action of full QCD at finite chemical potential. Secondly, there could be intrinsic problems in the lattice formulation of fermions (possibly associated with fermion doubling) and chemical potential which would survive an unquenched treatment.
Further studies[6] of the quenched theory on larger lattices found similar behaviour. However, a recent study [2 [5] of the quenched theory which measured the condensate and the pion and nucleon masses, did find that, at intermediate and strong coupling, the theory is sensitive to the baryon mass but that it is pathological for $\mu>\frac{m_{\pi}}{2}$.

From an analytical study of the eigenvalues of the fermionic propagator matrix Gibbs [7] concluded that the eigenmodes of the propagator matrix (defined below) relate to the mass spectrum of the theory. The Gibbs argument, requires the calculation of the hadronic spectrum on replicated lattices, i.e. lattices, with periodic boundary conditions on the gauge fields, which have been strung together $d$ times in the time direction. He considered the limit $d \rightarrow \infty$ in order to replace finite sums with contour integrations. The procedure is justifiable at zero temperature. The expression obtained for the inverse of the duplicated fermion matrix $G\left(t_{1}, t_{2}\right)$ is

$G\left(t_{1}, t_{2}\right)=\sum A_{a} \lambda_{a}^{t_{1}-t_{2}}$

where $A_{a}$ are the amplitudes (which can be related to the eigenvectors of the propagator matrix) and $\lambda_{a}$ are the associated eigenvalues. The form of the inverse shows that the exponential decay at large separation is controlled by the eigenvalues $\lambda_{a}$ : thus we see that the eigenvalue spectrum calculated on isolated configurations should contain poles in correspondence to the physical masses. In particular, the smallest mass state can clearly be extracted from the lowest eigenvalues. This state is obtained by the squaring the propagator matrix, and defines the pion mass in QCD. This implies that in the quenched theory $\langle\bar{\psi} \psi\rangle$ is controlled by the pion mass.

It has also been suggested by several authors that the coincidence of the onset of the chiral symmetry restoration with one half of the pion mass might only have been a numerical accident, the correct relationship being $\mu_{\text {onset }}=\frac{m_{N}}{3}-\Delta$ where $\Delta$ is the contribution of the nuclear binding energy. If this scenario were true then the problems with finite density simulations would not be too serious. This conjecture has been tested 
5. However this work confirmed the onset at $\mu=\frac{m_{\pi}}{2}$.

Recent work by Stephanov [8] using the random matrix method has provided some insight into this particular problem by demonstrating that at nonzero $\mu$ the quenched theory is not a simple $n_{f} \rightarrow 0$ limit of a theory with $n_{f}$ quarks but it is the $n_{f} \rightarrow 0$ limit of a theory with $n_{f}$ quarks and $n_{f}$ conjugate quarks and therefore inappropriate to QCD.This explanation implies that the phase of the determinant is important for a simulation of full QCD at finite $\mu$. In the quenched model the early onset for the number density would also correspond to the restoration of chiral symmetry because of the simultaneous occurrence of quarks and conjugate quarks in the system whereas the inclusion of dynamical fermions would result in a rearrangement of the eigenvalues such that the chiral symmetry would be restored at $\mu_{c} \simeq \frac{m_{B}}{3}$ as expected.

\section{Methods for Simulating QCD at $\mu \neq 0$ with Dynamical Fermions}

The pathologies of quenched studies of the chiral phase transition at finite density indicate that a correct implementation of dynamical quarks in the simulations is essential to the physics of the model.

\subsection{The Glasgow Method}

The problem caused by the non-Hermitian nature of the fermion matrix at $\mu \neq 0$ can be circumvented by a method which involves the expansion of the grand-canonical partition function $(\mathrm{GCPF})$ in powers of the fugacity variable $e^{\mu / T}$.

The GCPF can be expressed as an ensemble average of the fermionic determinant normalised with respect to the fermionic determinant at $\mu=$ 0 .

$$
\mathcal{Z}=\frac{\int[d U]\left[d U^{\dagger}\right]|M(\mu)| e^{-S_{g}\left[U, U^{\dagger}\right]}}{\int[d U]\left[d U^{\dagger}\right]|M(\mu=0)| e^{-S_{g}\left[U, U^{\dagger}\right]}}
$$

where $S_{g}$ is the pure gauge action and $M$ is the Kogut-Susskind fermion matrix.

This means that the probability measure is well-defined and standard Hybrid Monte-Carlo algorithms can be applied. The GCPF is then given by the ensemble average

$\mathcal{Z}=\left\langle\frac{|M(\mu)|}{|M(\mu=0)|}\right\rangle_{\mu=0}$

The Kogut-Susskind fermion matrix has the structure:

$2 i M=G+2 i m+\hat{V} e^{\mu}+\hat{V}^{\dagger} e^{-\mu}$.

in which $G$ contains all the spacelike gauge links and the bare quark mass and $\hat{V}\left(\hat{V}^{\dagger}\right)$ all the forward (backward) timelike links.

It then follows that, on a lattice of size $n_{s}^{3} n_{t}$, the determinant of $M$ is given by [7]

$|2 i M|=e^{3 \mu n_{s}^{3} n_{t}}\left|P-e^{-\mu}\right|$

where the propagator matrix $P$ is given by

$P=\left(\begin{array}{cc}-(G+2 i m) \hat{V} & \hat{V} \\ -\hat{V} & 0\end{array}\right)$

with its inverse given by

$P^{-1}=\left(\begin{array}{cc}0 & -\hat{V}^{\dagger} \\ \hat{V}^{\dagger} & -\hat{V}^{\dagger}(G+2 i m)\end{array}\right)$

From the structure of the propagator matrix and its inverse, it is easily shown that its eigenvalues have the symmetry that if $\lambda$ is an eigenvalue then so is $1 / \lambda^{\dagger}$. In addition, since the matrix $\hat{V}$ factors, the eigenvalues have a $Z\left(n_{t}\right)$ symmetry. As a consequence of this symmetry, the characteristic polynomial for $P$ is a polynomial in $e^{\mu n_{t}}$ with $6 n_{s}^{3}+1$ complex coefficients. Since the probability for a given configuration of gauge fields to appear in our ensemble is equal to that of its complex conjugate their imaginary parts will average to zero. We impose this symmetry in the simulations described below. As a consequence, the characteristic polynomial from a configuration (averaged with its complex conjugate) is invariant under $\mu \rightarrow-\mu$ and has the form:

$$
\sum_{n=-3 n_{s}{ }^{3}}^{3 n_{s}{ }^{3}} b_{|n|} e^{n \mu n_{t}}
$$


The chemical potential dependence of the GCPF is then given explicitly by

$$
\begin{aligned}
\mathcal{Z} & =\sum_{n=-3 n_{s}{ }^{3}}^{3 n_{s}{ }^{3}}\left\langle b_{|n|}\right\rangle e^{n \mu n_{t}} \\
& =\sum_{n=-3 n_{s}{ }^{3}}^{3 n_{s}{ }^{3}} e^{-\left(\epsilon_{n}-n \mu\right) / T}
\end{aligned}
$$

with $e^{-\epsilon_{n} n_{t}}=<b_{|n|}>$

By measuring the $\epsilon_{n}$ in the simulations we can readily obtain the fermion number density, $\rho=\langle n\rangle$ and the energy density, $E=<\epsilon_{n}>$.

The relative values of the canonical partition functions can be used to characterize the properties of the system. In particular the relative value of the triality non-zero coefficients give an indication of whether the system is in the confined or deconfined regime. In the confined sector the ensemble average of the triality non-zero coefficients should tend to zero as the statistics increase. We see strong evidence for this behaviour is seen in the lattice QCD simulations described below.

We also consider a description of the system in terms of the canonical partition functions for fixed particle number. This technique allows us to include only those triality zero coefficients which, at the end of the simulation, are positive and have a reasonable error. The chemical potential as a function of the baryon number density is obtained from the local derivative of the energy, $\epsilon_{n}$, of the state with $n$ fermions with respect to $n$.

$\mu(\rho)=\frac{1}{3 n_{s}^{3}} \frac{\partial \epsilon_{n}}{\partial \rho}$

where $\rho$ is the fermion density, $\frac{n}{3 n_{s}^{3}}$.

\subsection{Lee-Yang Zeros}

According to the theorems of Lee and Yang 9 the phase structure of a simulated system can be determined by studying the zeros of the GCPF. The zeros correspond to singularities in the thermodynamic potential. We study the zeros of the GCPF in the complex chemical potential (or fugacity plane) which are given by $Z(\mu)=0$. According to the Lee-Yang theorem as the system volume tends to infinity, the zeros will converge towards the critical value in the physical domain (real axis) of the chemical potential.

\subsection{Alternative Methods}

The Glasgow method involves an ensemble generated at $\mu=0$. This may introduce systematic errors due to insufficient overlap when measuring observables at $\mu$ significantly greater than the onset of non-zero number density. An alternative method involves generating the ensemble either with respect to the absolute value of $|M(\mu)|$ or with respect to the modulus of its real part 10,11 . In these simulations it is necessary to measure an additional observable related to the average sign of the determinant. For example, updating with respect to the modulus gives the observable $\mathcal{O}$ as

$\mathcal{O}=\frac{\left\langle\mathcal{O} e^{i \phi}\right\rangle_{|M|}}{\left\langle e^{i \phi}\right\rangle_{|M|}}$

where $\phi$ is the phase of the determinant. Simulations at a fixed quark mass using these methods are well behaved at low and high chemical potentials but for $\frac{m_{\pi}}{2} \leq \mu \leq \approx \frac{m_{p}}{2}$ the phase $\phi$ fluctuates violently from configuration to configuration and $\left\langle e^{i \phi}\right\rangle$ becomes very small and is not measurable. This is the region of $\mu$ in which critical behaviour is expected.

Gocksh 12 used a spectral density method by binning the phase $\phi$ and measuring $\rho(\phi)$. On a $2^{4}$ lattice, his results for $\mu_{c}$ are in broad agreement with meanfield.

\section{The Static Quark Model}

Blum, Hetrick and Toussaint 13 have recently studied numerical simulations of lattice QCD in the limit that the quark mass and chemical potential are simultaneously made large. In that limit, the quark mass and chemical potential appear only in the ratio $\left(2 m a / e^{\mu a}\right)^{n_{t}}$ and the propagator matrix becomes

$P=\left(\begin{array}{cc}-2 i m \hat{V} & \hat{V} \\ -\hat{V} & 0\end{array}\right)$.

The corresponding fermion determinant is complex but trivial to evaluate which allows generation of very high statistics in their measurements and determine $\left\langle e^{i \phi}>\right.$ to sufficient accuracy.

For quenched QCD the high temperature transition is first order and they expected this behaviour to extend into the interior of the $T$ - 
$\mu$ phase diagram. However their simulations showed that this transition becomes a smooth crossover at very small density (possibly for any nonzero density) and that, at low enough temperature, chiral symmetry remains broken at all densities.

Of course, as the authors point out,it is not at all clear that the static approximation has anything to do with real QCD. However, it is relevant that unexpected results follow in this simple model.

\section{Lattice QCD at Strong Coupling}

The strong coupling limit of QCD provides us with an important testing ground for lattice Monte-Carlo simulations at finite density because we can compare our results with the analytic predictions of mean-field theory. No such analytic predictions are available in the scaling region (i.e. intermediate coupling). At infinite coupling the mean-field method predicts a first order transition at chemical potential $\mu_{m f}$ where

$\mu_{m f}=\frac{1}{r} \sinh ^{-1}\left(\lambda_{0} r\right)-\frac{\lambda_{0}{ }^{2}}{(d-1) r}$

where

$\lambda_{0}=\frac{1}{r \sqrt{2}}\left(\sqrt{1+(d-1)^{2} r^{4}}-1\right)^{1 / 2}$

for a lattice with $d$ space-time dimensions where $r=n_{t} / n_{s}$.

The mean-field baryon and pion masses are given by

$$
\begin{aligned}
& m_{B}=\ln {\left[\frac{1}{2} c^{3}+\sqrt{1+\frac{1}{8} c^{6}}\right] } \\
& m_{\pi}=\ln \left[1+\frac{1}{2}\left(c^{2}-2 d\right)\right. \\
&\left.+\sqrt{\left(c^{2}-2 d\right)+\frac{1}{4}\left(c^{2}-2 d\right)^{2}}\right]
\end{aligned}
$$

where

$c=\left(m+\sqrt{2 d+m^{2}}\right)$
Note that $\mu_{m f}$ does not correspond to the meanfield baryon threshold $m_{B} / 3$.

It has been argued that the discrepancy is related to the binding energy of nuclear matter which is large at infinite coupling. Bilic et al.14 demonstrated explicitly that $1 / g^{2}$ corrections diminish the discrepancy between $\mu_{m f}$ and the mass of the lightest baryonic state divided by $N$ (the number of colours). The mean-field pion thresholds and $\mu_{m f}$ are not well separated for bare quark masses $m a>0.5$.

Monte-Carlo SU(3) simulations 15 using the monomer-dimer algorithm whereby the partition function is represented in terms of monomers, dimers and baryonic loops estimate a critical chemical potential, $\mu_{c}$ which is in agreement with $\mu_{m f}$. This algorithm involves integrating out the gauge fields exactly and subsequently integrating the quark fields. Hybrid Monte-Carlo techniques integrate the quark fields prior to the gauge fields. The monomer-dimer algorithm cannot be used to explore small bare quark masses $m<0.1$ and is restricted to the infinite coupling regime where the quarks are point-like.

\subsection{Strong Coupling using the Glasgow Method}

Consider again the expression for the GCPF

$\mathcal{Z}=\sum_{n=-3 n_{s}{ }^{3}}^{3 n_{s}{ }^{3}} e^{-\left(\epsilon_{n}-n \mu\right) / T}$

The zeros of this polynomial are the Lee-Yang zeros of $Z$ in the complex fugacity plane (or equivalently the complex $e^{\mu}$ plane). In the dynamical theory these zeros play a role analogous to that of the eigenvalues of $P$ in the quenched theory when calculating the number density.

$\mathrm{Z}$ can be rewritten as

$Z=e^{3 n_{s}^{3} \mu / T} \prod_{i=1,6 n_{s}{ }^{3}}\left(z-\alpha_{i}\right)$

where $z=e^{-\mu / T}$ and the $\alpha_{i}$ are the zeros of the averaged characteristic polynomial for $P$.

On each configuration

$$
\operatorname{det}(M)=e^{3 n_{s}^{3} \mu / T} \prod_{i=1,6 n_{s}{ }^{3}}\left(z-\lambda_{i}\right)
$$


where the $\lambda_{i}$ are the eigenvalues of $P$. Since $Z=\left\langle\frac{\operatorname{det} M(\mu)}{\operatorname{det} M(\mu=0)}\right\rangle_{\mu=0}$ we can interpret the zeros of the partition function as the proper ensemble average of the eigenvalues of the fermionic propagator matrix. One obvious constraint on the distribution of the zeros is that in the confined sector we expect to see a $Z(3)$ symmetry arising from the triality non-zero coefficients averaging to zero so that only canonical partition functions containing multiples of three quarks contribute to the thermodynamics. The $Z(3)$ symmetry constrains the phases of the zeros but not their modulus.

We calculate the fermion number density, $n_{q}$ from

$$
<n_{q}>=\frac{T}{V} \frac{\partial \ln (Z)}{\partial \mu}
$$

Hence in the quenched theory the number density, $n_{q}$, is given by

$n_{q}=1+<\frac{1}{3 V} \sum_{-3 V}^{3 V} \frac{z}{\lambda_{i}-z}>$.

where the $\lambda_{i}$ are the eigenvalues of $P^{n_{t}}$. Using the generalized boundary conditions arising from considering a configuration on a replicated lattice one [7] can convert the sum to a contour integral and obtain, for each configuration

$n_{d}=1 / V \sum_{1<\left|\lambda_{i}\right|<e^{\mu / T}} 1$.

In the dynamical theory the number density, $n_{d}$, is given by

$n_{d}=1+\frac{1}{3 V} \sum_{-3 V}^{3 V} \frac{z}{\alpha_{i}-z}$

where the $\alpha_{i}$ are the zeros of the averaged characteristic polynomial for $P$. Performing the contour integation as above gives

$n_{d}=1 / V \sum_{1<\left|\alpha_{i}\right|<e^{\mu / T}} 1$.

Thus if we consider plotting the zeros of the GCPF in the complex $e^{\mu}$-plane, any discontinuities in the number density will be associated with circular bands of increased density of zeros.

\subsection{Results at Strong Coupling}

We first consider, in the light of the above discussion concerning the roles of the eigenvalues and the zeros of the GCPF, their distributions in the complex $\mu$ and $e^{\mu}$ planes.
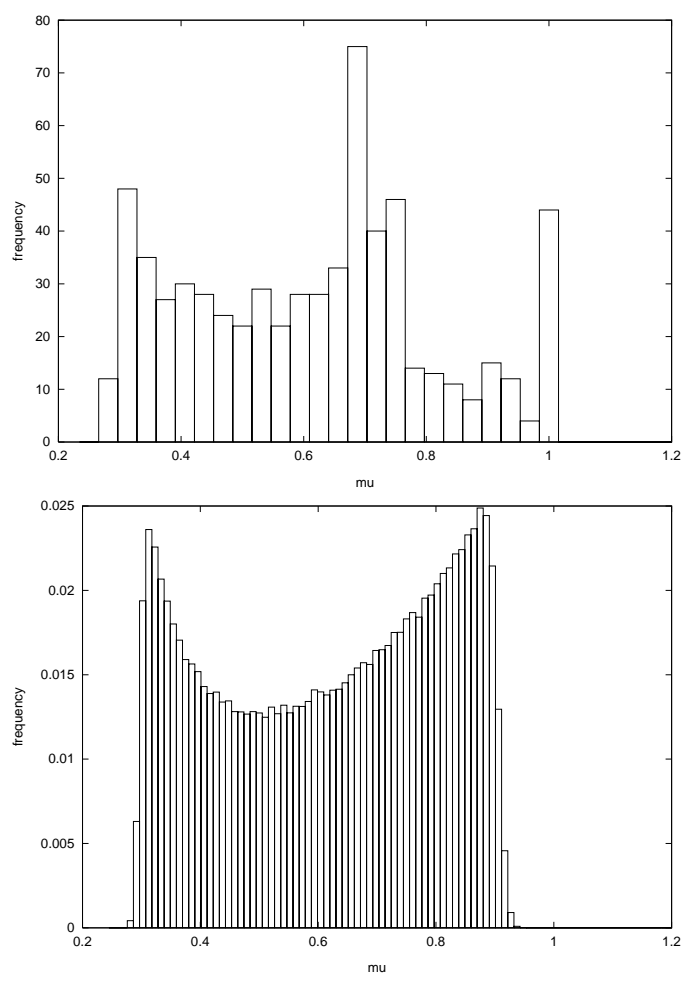

Figure 1. Histograms of the real parts of the eigenvalues and of the real part of the zeros in the complex $\mu$-plane at strong coupling and bare quark mass, $m a=0.08$ on a $6^{4}$ lattice.

Fig 11 contains a histogram of the distribution of the positive real parts of the eigenvalues in the complex $\mu$ plane. The distribution is obtained from 450 configurations on a $6^{4}$ lattice at a bare quark mass of 0.08 . There is a clear signal that the number density becomes non-zero at $\mu \approx 0.3$ which is consistent with $m_{\pi} / 2$. This is the quenched onset $\mu$. The lattice is filled at $\mu=0.95$. 
The analogous distribution of the zeros of the GCPF is also shown in Fig 1. These zeros are those of the polynomial obtained by averaging the characteristic polynomials of the propagator matrix over the same 450 configurations. There is still a clear signal that the number density becomes non-zero at the same onset $\mu$ as that of the quenched theory but a strong signal has developed via an intermediate peak in the distribution which is absent from that of the eigenvalues. As argued above, this band of increased density can be associated with a discontinuity in the number density.

This difference in distribution between the eigenvalues and the zeros is found at all other bare quark masses. Fig. 2 and Fig. 3 show our results at quark mass $m=0.05$ while Fig. 团and Fig. 孟 show results at $m=0.5$.

Figs. 6 and 7 show clearly the banded structure of the zeros distribution in the $e^{\mu}$ plane for two different lattice sizes and quark masses.

Fig. 8 compares the distribution of the real part of the zeros with the number density (on a $6^{4}$ lattice with $m=0.1$ ). Again the histogram for the zeros shows three distinct peaks: $\mu_{o} \simeq 0.3$ corresponding to the onset of net non-zero quark density; $\mu_{c} \simeq 0.7$ corresponding to the small discontinuity in the number density and the expected critical chemical potential; $\mu_{s} \simeq 1.0$ corresponding to the lattice saturation point. Comparison of the two plots in this figure shows that the derivative of the number density correlates well with the density of states (frequency histogram).

Fig. 9 gives an overview of the location of $\mu_{o}$ and $\mu_{c}$ for six quark masses in the range $m a=0.05$ to 1.5 . The scaling of the central peak of the histogram is in remarkable agreement with the mean-field prediction calculated form eqn.13 and is consistent with the critical $\mu$ predicted by the monomer-dimer simulations. The peak corresponding to $\mu_{o}$ is consistent with the mean-field pion threshold for the smaller quark masses but significantly lower for $m a=1.0,1.5$.

\section{Lattice QCD at Intermediate Coupling}

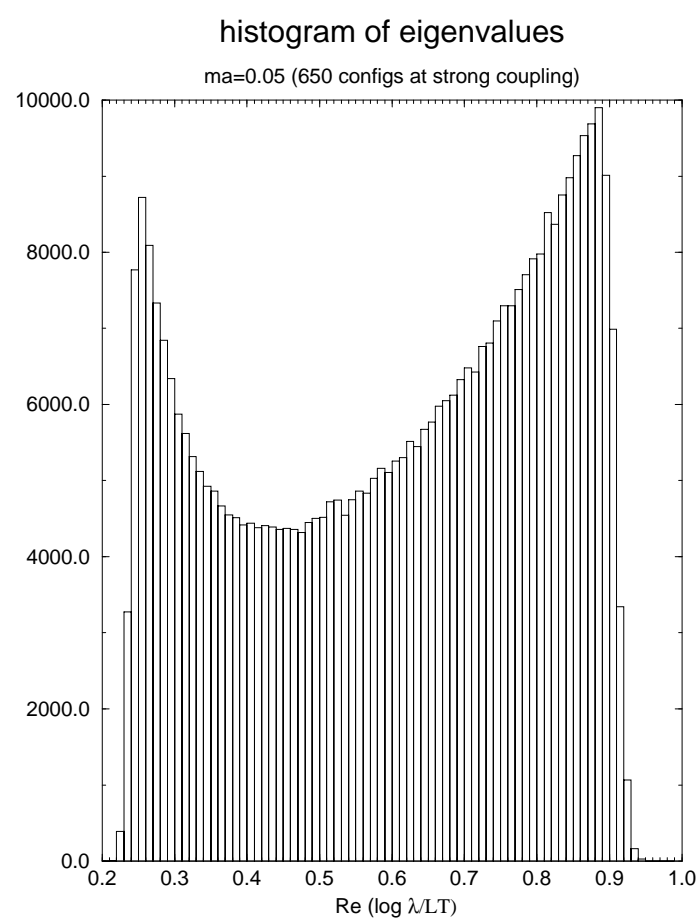

Figure 2. Histogram of the real parts of the eigenvalues in the complex $\mu$-plane at strong coupling and bare quark mass, $m a=0.05$ on a $6^{4}$ lattice.

\section{1. $Z(3)$ Tunnelling}

In the pure gauge theory we have $N_{c}(=3)$ equivalent vacua related by $Z\left(N_{c}\right)$ rotations. Tunnelling between the different $Z(3)$ vacua is much more probable in the confined sector than in the deconfined sector. Since the pure-gauge action as well as the integration measure are invariant under the $Z(3)$ transformation, the GCPF can also be written as:

$\mathcal{Z}(\mu)=\frac{\int[d U]\left[d U^{\dagger}\right] \operatorname{det} M\left(\mu+z_{3} / n_{t}\right) e^{-S_{g}\left[U, U^{\dagger}\right]}}{\int[d U]\left[d U^{\dagger}\right] \operatorname{det} M(\mu=0) e^{-S_{g}\left[U, U^{\dagger}\right]}}(27)$

Averaging over the three $Z(3)$ vacua would eliminate the triality non-zero coefficients and, since $Z(0)=1$, the sum of the triality zero coefficients should tend to 1 . As Fig. 10 shows, at $\beta=5.1$ and $m=0.01$, we obtained a clear signal for $\mathrm{Z}(3)$ tunnelling. The sum of the triality zero coefficients 
Histogram of distribution of zeros

$\mathrm{ma}=0.05$

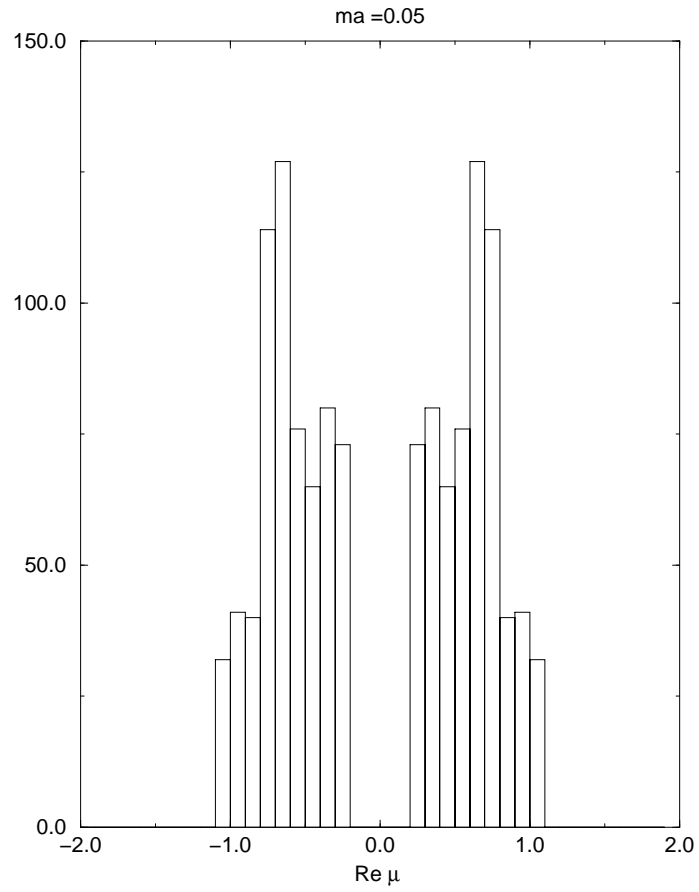

Figure 3. Histogram of the real parts of the zeros in the complex $\mu$-plane at strong coupling and bare quark mass, $m a=0.05$ on a $6^{4}$ lattice.

shows a strong tendency to average to 1 whilst the sum of the triality one (and two) coefficients tends to zero. This is consistent with simulating in the confined phase at $\mu=0$ as expected at this coupling and quark mass.

\subsection{Simulations on $6^{4}$ and $8^{4}$ lattices at in- termediate coupling}

We have investigated 16] the chemical potential (the onset $\mu$ ) required to make the level with 3 quarks equally probable with that of the zero quark level, i.e. $\mu_{\text {onset }}=\left(\epsilon_{3}-\epsilon_{0}\right) / 3$. This ad-hoc definition takes into account that it is these energy levels which are most accurately determined and allows errors to be estimated directly.

Fig. 11 shows the dependence of the onset $\mu$ on the quark mass and on the square root of the quark mass on a $6^{4}$ lattice at gauge coupling

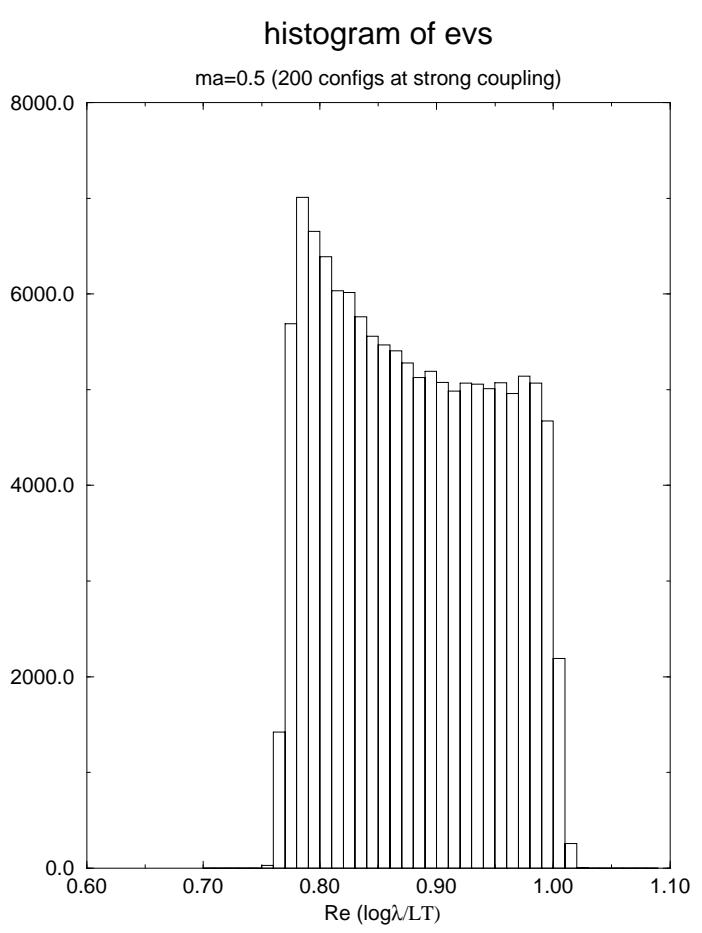

Figure 4. Histogram of the real parts of the eigenvalues in the complex $\mu$-plane at strong coupling and bare quark mass, $m a=0.5$ on a $6^{4}$ lattice.

$\beta=5.1$. As at strong coupling, there is a strong signal that this onset $\mu$ is dependent on the mass of a Goldstone boson for quark mass $m>0.01$. (On a $6^{4}$ lattice, at this $\beta$, the system becomes 'deconfined' at some $m<0.01$. We have checked this behaviour by determining its Lee-Yang zeros in the complex $m$-plane and they are complex with non-zero real part $<0.01$.)

Fig. 12 shows the distribution of the zeros found on a $8^{4}$ lattice at the same $\beta$ and quark mass 0.01 in the $e^{\mu}$ plane. The zeros associated with the onset $\mu$ lie on the arc of a circle (this circular pattern is also observed in the strong coupling results described above). Again the distribution is consistent with a number density becoming non-zero at a small $\mu$. Also we see a circular band of zeros developing at $0.7<\mu<0.9$. 


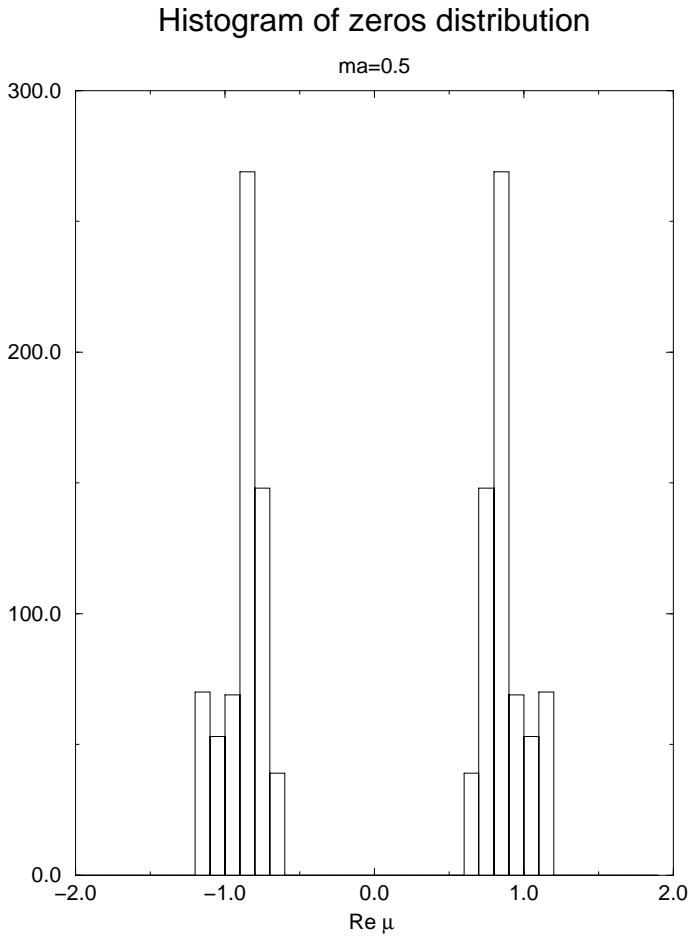

Figure 5. Histogram of the real parts of the zeros in the complex $\mu$-plane at strong coupling and bare quark mass, $m a=0.5$ on a $6^{4}$ lattice.

We believe that this is a signal for the physical $\mu_{c}$.

\section{The U(1) Gross-Neveu Model}

\subsection{The Lattice Formulation}

In the above simulations of $\mathrm{QCD}$, the initial onset of the critical behaviour at non-zero $\mu$ was controlled by the pion mass rather than that of the lightest baryon. To check if this is an artifact arising from measuring our observables on an ensemble generated at $\mu=0$ we have investigated the above methods in a study of the Gross-Neveu model at finite density. The lattice formulation of this model is described in ref.[17]

The lattice action for the bosonized Gross-

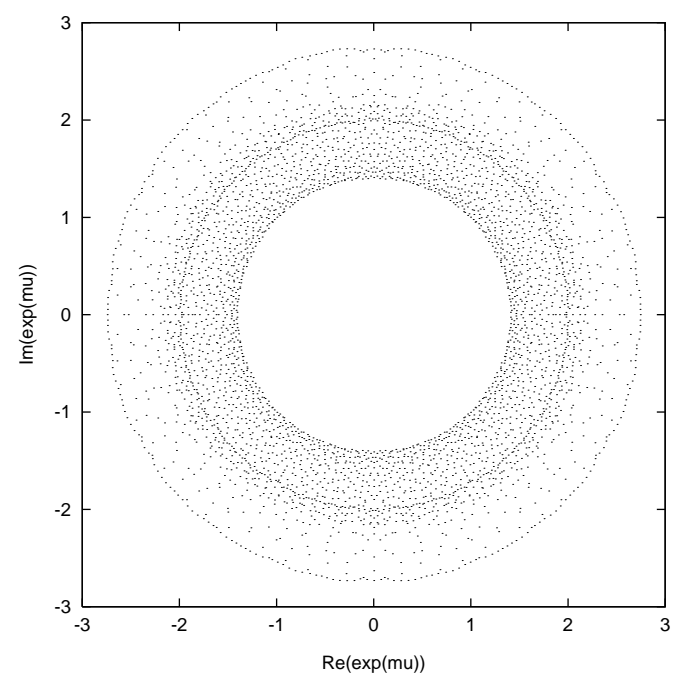

Figure 6. Zeros in the $e^{\mu}$ plane for $m=.095$, $6^{4}$ lattice. The critical line is the thin line inside the denser region $e^{\mu}=e^{\mu_{c}}$

Neveu model with $\mathrm{U}(1)$ chiral symmetry is

$$
\begin{aligned}
S & =\sum_{i=1}^{N_{f} / 4}\left[\sum_{x, y} \bar{\chi}_{i}(x) \mathcal{M}_{x, y} \chi_{i}(y)\right. \\
& +\frac{1}{8} \sum_{x} \bar{\chi}_{i}(x) \chi_{i}(x)\left(\sum_{<\tilde{x}, x>} \sigma(\tilde{x})\right. \\
& \left.\left.+i \varepsilon(x) \sum_{<\tilde{x}, x>} \pi(\tilde{x})\right)\right] \\
& +\frac{N_{f}}{8 g^{2}} \sum_{\tilde{x}}\left(\sigma^{2}(\tilde{x})+\pi^{2}(\tilde{x})\right) .
\end{aligned}
$$

Here, $\chi_{i}$ and $\bar{\chi}_{i}$ are complex Grassmann-valued staggered fermion fields defined on the lattice sites, the auxiliary scalar and pseudoscalar fields $\sigma$ and $\pi$ are defined on the dual lattice sites, and the symbol $\langle\tilde{x}, x\rangle$ denotes the set of 8 dual sites $\tilde{x}$ adjacent to the direct lattice site $x . N_{f}$ is the number of physical fermion species.

The fermion kinetic operator $\mathcal{M}$ is given by

$\mathcal{M}_{x, y}=\frac{1}{2}\left[\delta_{y, x+\hat{0}} e^{\mu}-\delta_{y, x-\hat{0}} e^{-\mu}\right]$ 


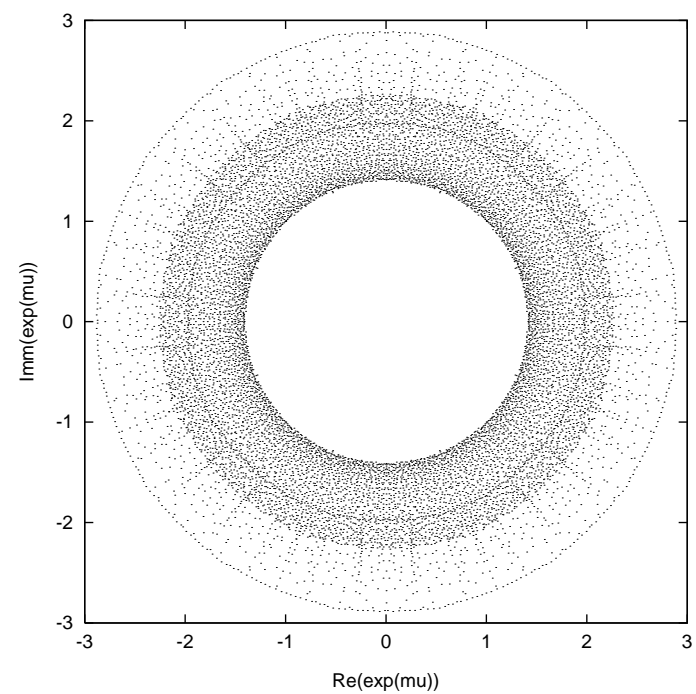

Figure 7. Zeros in the $e^{\mu}$ plane for $m=.18^{4}$ lattice.

$$
\begin{aligned}
& +\frac{1}{2} \sum_{\nu=1,2} \eta_{\nu}(x)\left[\delta_{y, x+\hat{\nu}}-\delta_{y, x-\hat{\nu}}\right] \\
& +m \delta_{y, x},
\end{aligned}
$$

where $m$ is the bare fermion mass, $\mu$ is the chemical potential, and $\eta_{\nu}(x)$ are the Kawamoto-Smit phases $(-1)^{x_{0}+\cdots+x_{\nu-1}}$. The symbol $\varepsilon(x)$ denotes the alternating phase $(-1)^{x_{0}+x_{1}+x_{2}}$.

This lattice model (at non-zero lattice spacing) has the symmetry: $\mathrm{U}\left(N_{f} / 4\right)_{V} \otimes \mathrm{U}\left(N_{f} / 4\right)_{V} \otimes$ $\mathrm{U}(1)_{A}$. It is the $\mathrm{U}(1)_{A}$ symmetry which is broken, either spontaneously by the dynamics of the system, or explicitly by a bare fermion mass. We simulated the $N_{f}=12$ model corresponding to three lattice species.

The expansion of this GCPF is very similar to that described above for standard QCD. At finite density the Dirac fermion matrices $M$ and $\hat{M}$ are given by:

$$
\begin{aligned}
2 i M_{x y}(\mu) & =Y_{x y}+G_{x y}+V_{x y} e^{\mu}+V_{x y}^{\dagger} e^{-\mu} \\
-2 i \hat{M}_{x y}(\mu) & =Y_{x y}^{\dagger}+G_{x y}+V_{x y} e^{\mu}+V_{x y}^{\dagger} e^{-\mu} .
\end{aligned}
$$

where $Y=2 i\left(m_{q}+\frac{1}{8} \sum_{<x, \tilde{x}>}(\sigma(\tilde{x})+i \epsilon \pi(\tilde{x}))\right) \delta_{x y}$.

The determinants of these fermion matrices are
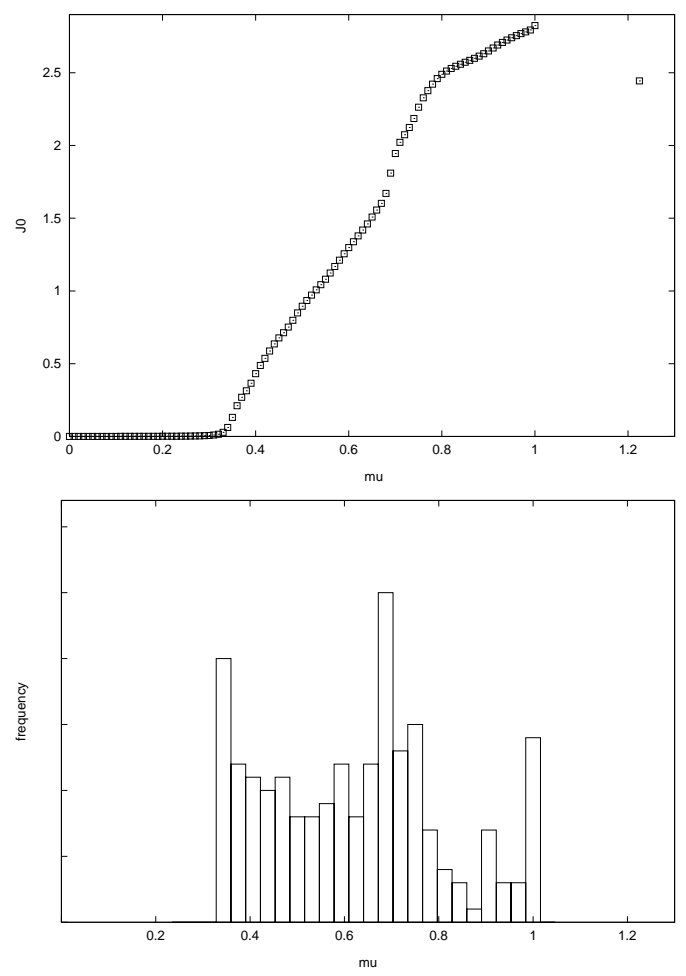

Figure 8 . The number density at quark mass $m_{q}=.1$ (upper) on a $6^{4}$ lattice at strong coupling. The behaviour of the number density is reflected in the histogram of the real parts of the zeros in the complex $\mu$-plane shown in the lower portion of the figure.

related to that of the propagator matrix

$P=\left(\begin{array}{cc}-G V-Y V & V \\ -V & 0\end{array}\right)$

by $\operatorname{det}(2 i M)=e^{3 \mu n_{s}^{3} n_{t}} \operatorname{det}\left(P-e^{-\mu}\right)$ and $\operatorname{det}(2 i \hat{M})=e^{3 \mu n_{s}^{3} n_{t}} \operatorname{det}\left(\left(P^{-1}\right)^{\dagger}-e^{-\mu}\right)$.

As for standard QCD, determination of the eigenvalues of $P^{n_{t}}$ gives the expansion for the GCPF:

$Z=\sum_{n=-2 n_{s}^{2}}^{2 n_{s}^{2}}<b_{|n|}>e^{n \mu n_{t}}$ 


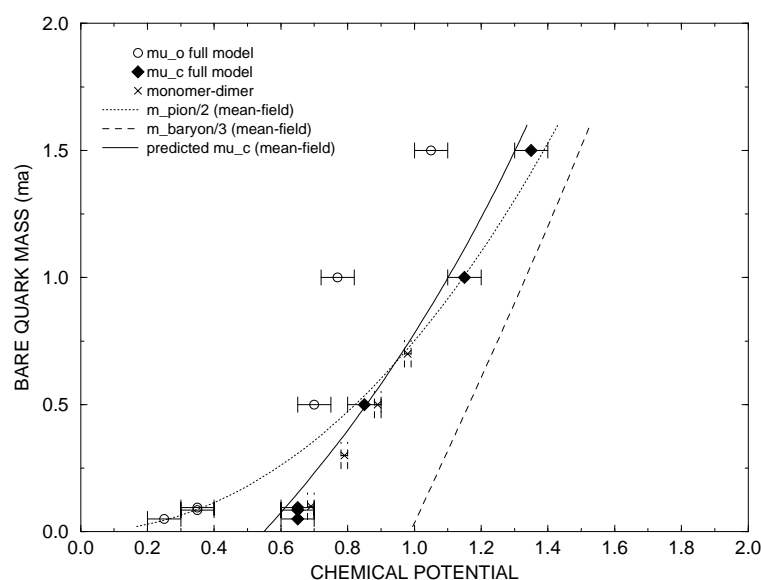

Figure 9. Summary of the results for the critical point $\mu_{c}$, and current onset $\mu_{o} . \mu_{c}$ follows the prediction of the mean field analysis of ref.[4]. The onset is close to half the pion mass at small mass, and lower for $m_{q}>.5$. The data for the pion mass are from ref. [7]

$$
=\sum_{n=-2 n_{s}{ }^{2}}^{2 n_{s}{ }^{2}} e^{-\left(\epsilon_{n}-n \mu\right) / T} .
$$

\subsection{Results}

This model has a massless pion and its fermion determinant is real and non-negative even when $\mu \neq 0$. Therefore standard Monte-Carlo algorithms can be used to study its critical behaviour as a function of $\mu$.

In particular, at an inverse four-fermion coupling of 0.5 and bare fermion mass $m=0.01$, the theory has a critical chemical potential $\mu_{c}=$ $0.725(25)$ at which the chiral symmetry is restored. Note, the Gross-Neveu model is nonconfining and the gauge fields do not enter into the dynamics.

We performed simulations developing the ensemble at $\mu=0$ to confirm that Eqn. 5 does give an extrapolation which predicts the correct critical behaviour. The number density for a $16^{3}$ lattice at the above coupling and fermion mass is shown in Fig. 13. The chemical potential at which the number density, $\langle n\rangle$, becomes non -
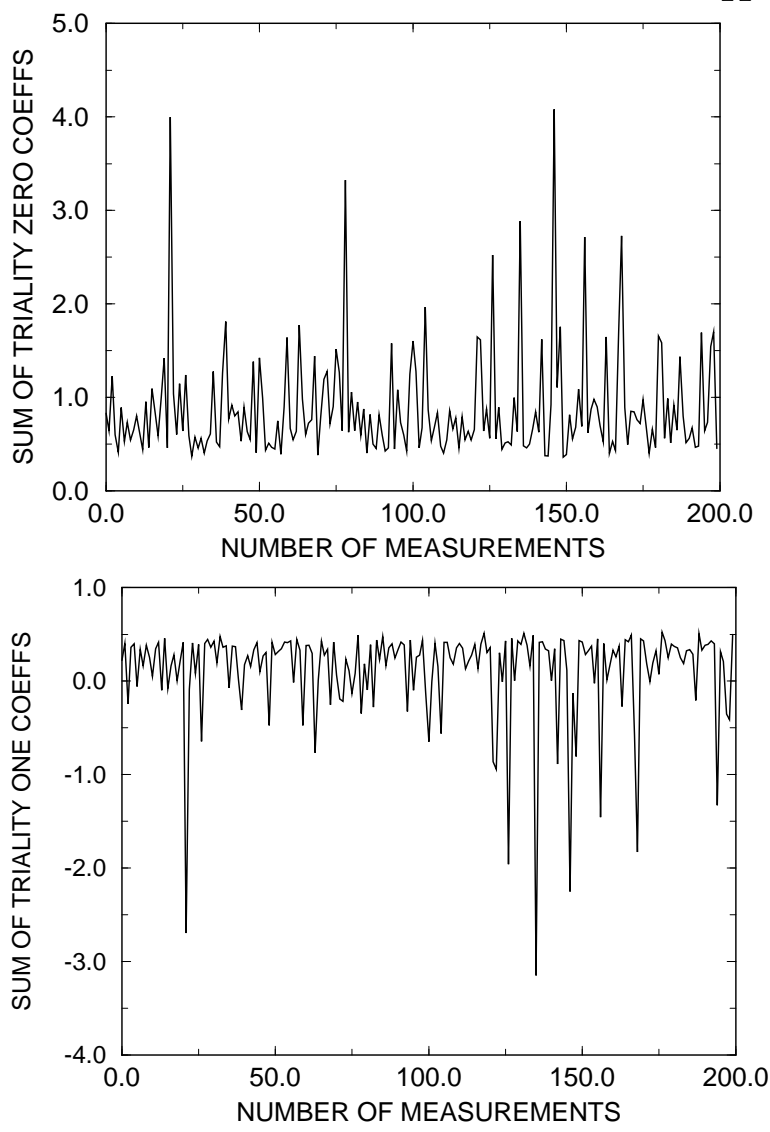

Figure 10. The HMC time evolution of the expansion coefficients on an $8^{4}$ lattice at $\beta=5.1$ and $m a=0.01$. The behaviour of the triality 2 coefficients is similar to that of the triality 1 coefficients.

zero, $\mu_{o}$, is correctly predicted. Comparison with the exact simulation[17] (in which generation of the ensemble was at the $\mu$ at which the measurement was made) shows that at $\mu>\mu_{o}$ the number density is underestimated and the discontinuity in $\langle n\rangle$ is absent. The Lee-Yang zeros in the complex $\mu$ plane are the zeros of Eqn. 5 and should signal the critical $\mu$ associated with the chiral transition transition. They are shown in Fig. 14 There is a line formed by 6 zeros, two of which are isolated from the rest of the distribution. The line intersects the real $\mu$ axis at $\mu=0.72$, in agreement with the critical $\mu$ found by the exact method.

Hence we find that the onset $\mu$ found via the 

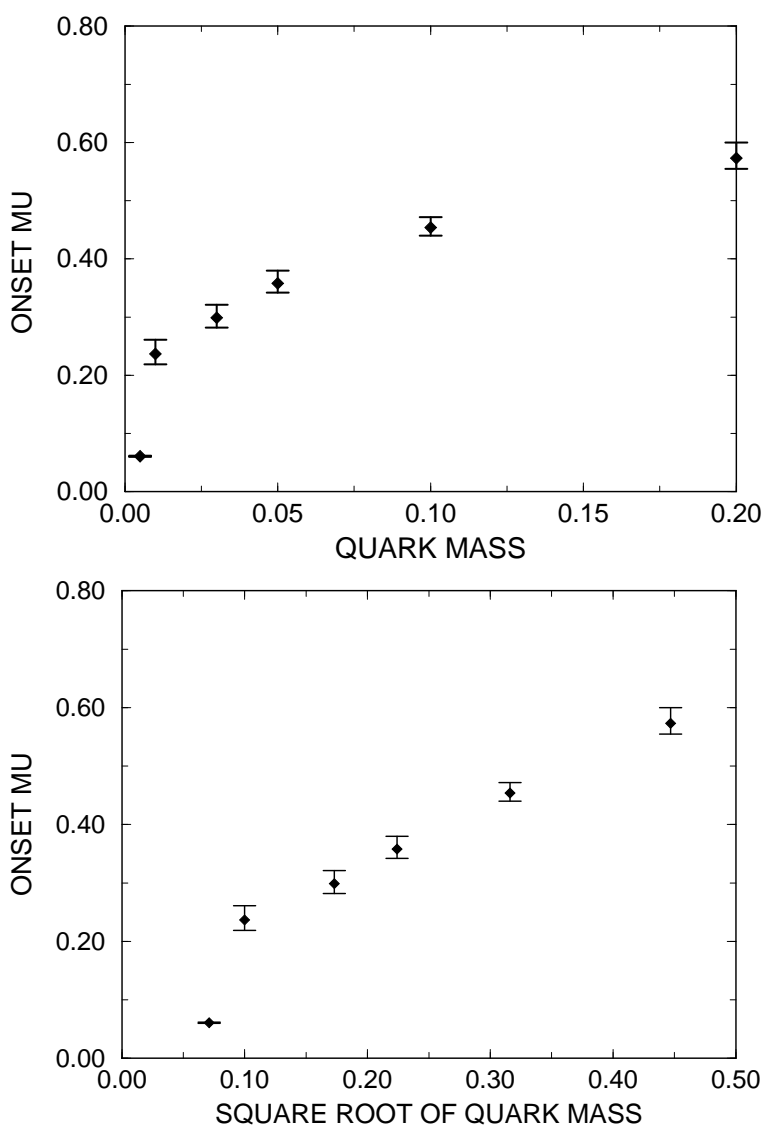

Figure 11. The quark mass dependence of the onset $\mu\left(\beta=5.1\right.$ on a $6^{4}$ lattice $)$.

number density is not controlled by the Goldstone boson, and the critical $\mu$ is given by the distribution of the Lee-Yang zeros.

Fig. 13 also shows the number density obtained from an ensemble generated at an update $\mu=0.8$, greater than $\mu_{c}$. In the chiral limit it is consistent with that of a free gas of massless fermions whereas the number density from the $\mu=0$ update is that of a free gas of massive fermions. The exact results show the transition between these two phases at $\mu_{c}$.

\section{Conclusions}

Simulation of standard lattice QCD at finite density seems to be plagued by the problems of the naive quenched version of the theory, namely

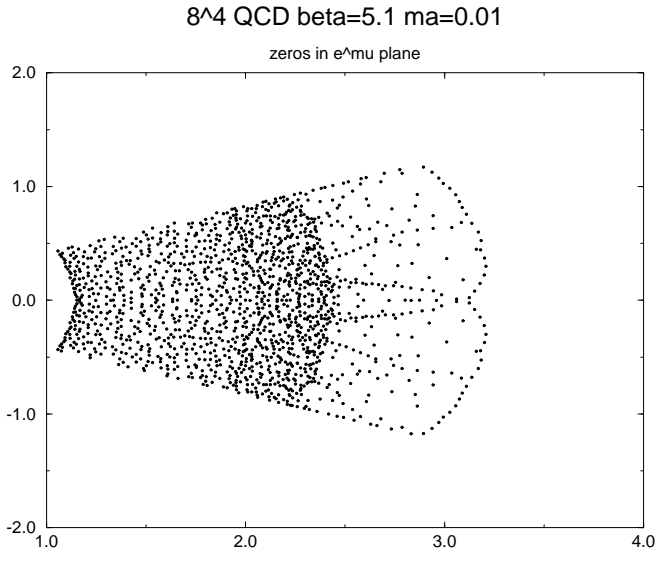

Figure 12. The Lee-Yang zeros on a $8^{4}$ lattice in the complex $e^{\mu}$ plane at $\beta=5.1$ and bare quark mass 0.01. Only one segment is shown but the intrinsic $Z\left(n_{t}=8\right)$ of Figs. 4 and 5. also applies here.

the chemical potential at which the fermion number begins to differ from zero is controlled by a Goldstone pion rather than by the lightest baryon.

Clearly questions must be answered:

Why do we continue to observe an onset chemical potential which scales with the pion mass? Is the band of zeros at higher $\mu$ a signal of the transition in the number density? Is this band associated with the deconfinement and chiral transitions? Do these two transitions occur at the same $\mu$ ?

The success of the monomer dimer approach (of use only at strong coupling) may be due to analytic integration first over the gauge fields, and then the fermion fields, giving an effective action with a reduced sign problem. Standard Hybrid Monte-Carlo algorithms integrate the fermion fields prior to the gauge fields. We are now attempting to understand why at strong coupling, we find an onset chemical potential, $\mu_{o}$, in addition to the expected signal at $\mu_{c}$.

Incomplete cancellation could arise from either limited statistics or from the reweighting procedure itself. Using an ensemble created at $\mu=0$, 


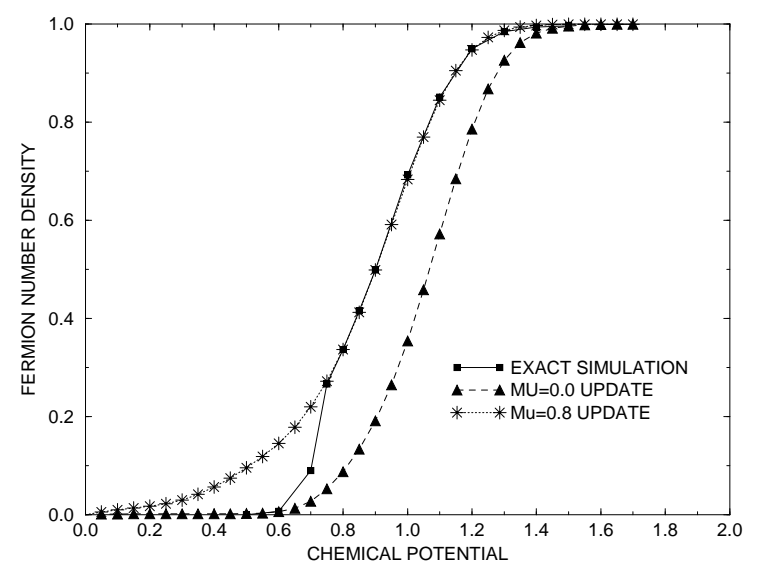

Figure 13. The fermion number density vs. the chemical potential, $\mu$, on a $16^{3}$ lattice at an inverse four-fermion coupling 0.5 and fermion mass 0.01

together with reweighting with respect to the fermion determinant at $\mu=0$ may not give sufficient overlap with an ensemble generated at $\mu \neq 0$ to correctly describe the true physics there. Although a limited increase in statistics revealed that the peak in the histogram of zeros corresponding to $\mu_{o}$ grew proportionally with the peak at $\mu_{c}$ it is still possible that the signal for $\mu_{o}$ will be cancelled out in the limit of infinite statistics once the $Z(3)$ invariance has been completely achieved.

Another solution may be to approach QCD by adding a perturbatively irrelevant four-fermion interaction term to the action and to study the critical nature of that theory as this additional term is weakened. This approach permits simulations directly in the chiral limit.

\section{Acknowledgements}

We would like to thank Misha Stephanov for very useful suggestions. This work was supported in part by the NSF through grant NSFPHY92-00148, by DOE, by PPARC through grant GR/K55554 and by NATO via grant CRG960002. Computations were performed on the C90s at

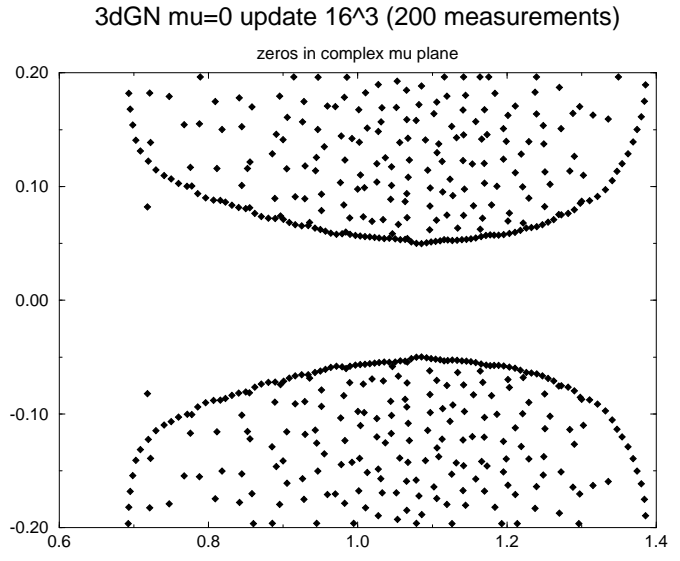

Figure 14. The Lee-Yang zeros in the complex $\mu$ plane for the Gross-Neveu model at an inverse four-fermion coupling 0.5 and fermion mass 0.01 on a $16^{3}$ lattice

NERSC and PSC.

\section{REFERENCES}

1. I.M. Barbour,N. Behilil, E. Dagotto, F. Karsch, A.Moreo, M.Stone, H. Wyld, Nuclear Physics B275 (FS17)(1986) 296.

2. J.B. Kogut, M.P. Lombardo and D.K. Sinclair, Phys. Rev. D51 (1995) 1282.

3. J.B. Kogut, H. Matsuoka, M. Stone, H.W. Wyld, S. Shenker, J. Shigemitsu and D.K. Sinclair, Nucl. Phys. B225 [FS], (1983) 93.

4. P. Hasenfratz and F. Karsch, Phys. Lett. B125 (1983) 308.

5. J.B. Kogut, M.P. Lombardo and D.K. Sinclair, Phys. Rev. D54 (1996) 2303.

6. C.T.H. Davies and E.G. Klepfish, Phys. Lett. B256 (1991) 68.

7. P.E. Gibbs, Phys. Lett. 172B (1986) 53.

8. M.A. Stephanov, Phys. Rev. Lett. 76 (1996) 4472.

9. C.N. Yang and T.D. Lee, Phys. Rev. 87 (1952) 104

T.D. Lee and C.N. Yang, Phys. Rev. 87 (1952) 410

10. A. Vladikas, Nucl. Phys.B (Proc. Suppl.) 4 
(1988) 322 .

11. D. Toussaint, Nucl. Phys. B (Proc. Suppl.) 17 (1990) 248.

12. A. Gocksh, Phys. Lett. B61 (1988) 2054.

13. T. Blum, J.E. Hetrick and D. Toussaint, Phys. Rev. Lett. 76 (1996) 1019.

14. N. Bilić, K. Demeterfi and B. Petersson, Nucl. Phys. B377, 651 (1992).

15. F. Karsch and K. Mütter, Nucl. Phys. B313 (1989) 541.

16. I.M. Barbour, S. Morrison and J.B. Kogut, hep-lat/9607057.

17. S. Hands, S. Kim and J.B. Kogut, Nucl.Phys. B442 (1995) 364. 